\title{
Circuito Venoso Cervical de la Llama (Lama glama)
}

\author{
Cervical Venous Circuit of the Llama (Lama glama)
}

Carlos Alberto Arzone; Gabriel Alejandro Sánchez; Ramiro Vidal Figueredo; Marcelo Acerbo \& Fernando Carlos Pellegrino

ARZONE, C. A.; SÁnCHEZ, G. A.; VIDAL FIGUEREDO, R. V.; ACERBO, M. \& PELlEGRINO, F. C. Circuito venoso cervical de la llama (Lama glama). Int. J. Morphol., 26(4): 849-852, 2008.

RESUMEN: La particular estructura de la región cervical de la llama (Lama glama) se traduce en numerosas diferencias anátomofuncionales respecto de otros mamíferos domésticos. Entre ellas merece destacarse un distintivo circuito venoso, cuya descripción es el objetivo de este trabajo. Para el presente estudio, se utilizaron diez llamas, machos, adultos y de aptitud lanera. Para resaltar las venas del cuello se procedió a la repleción de las mismas y, posteriormente, se disecaron los vasos utilizando técnicas e instrumental convencional. Se describe la morfología particular de un circuito venoso, en el cual las venas yugular externa, vertebral e intervertebrales son las principales protagonistas. El hecho destacado radica en las anastomosis establecidas entre las citadas venas, uniones dispuestas en forma metamérica, formando un circuito o by pass yugular-vertebral. La existencia de comunicaciones o shunts venosos, permite definir un circuito de circulación colateral derivando la sangre principalmente hacia la vena yugular externa. La longitud del cuello y los hábitos de pastoreo indican que esta disposición vascular es un hecho facilitador del retorno venoso, en cuanto a su dinámica y al control regional del flujo sanguíneo.

PALABRAS CLAVE: Venas cervicales; Anatomía Veterinaria; Camélidos sudamericanos.

\section{INTRODUCCIÓN}

Los camélidos sudamericanos conforman un pequeño grupo zoológico que incluye dos especies domésticas, la llama y la alpaca, y otras dos silvestres, la vicuña y el guanaco.

Además de ser una especie productiva, la llama se ha popularizado en los últimos años, incorporándose a zoológicos, parques y jardines, con propósitos educativos, ornamentales y de preservación. En la actualidad, su cría y producción se han extendido desde la región andina sudamericana hasta los Estados Unidos de América y Europa.

Acorde con esta tendencia, las ciencias básicas veterinarias se han aproximado al conocimiento de esta especie en las últimas tres décadas, pero con un enfoque poco sistemático que ha ocasionado una notable ausencia bibliográfica en determinados temas. Se suma a esto, el problema de que numerosas veces se ha inferido erróneamente que la bioestructura de la llama es similar a la de los rumiantes domésticos tradicionales.

Este trabajo realiza un aporte al conocimiento de la angiología de la región cervical de la llama, como base para futuras aplicaciones médico-quirúrgicas y zootécnicas. Se recurrirá constantemente a la anatomía comparada, cotejando las estructuras con aquellas presentes en bovinos, equinos, caninos, porcinos y también con los camellos y dromedarios. Estas últimas especies se han incluido debido a su cercanía taxonómica con la llama y a la abundante bibliografía existente sobre las mismas.

En los mamíferos domésticos, la vena yugular externa se forma por la unión de las venas linguofacial y maxilar en la región parotídea, a nivel del ala del atlas. Desde allí la vena sigue su curso caudalmente a lo largo del cuello, alojada en el surco yugular; en este recorrido la vena cruza la superficie lateral del músculo omohioideo -excepto en los carnívoros, en los cuales está ausente- que la separa de la arteria carótida común, el tronco vagosimpático y el nervio laríngeo recurrente. Cerca de la abertura craneal del tórax, la vena yugular recibe a las venas cervical superficial y a la cefálica, con las que forma un tronco común en el gato y a las que se suma la vena omobraquial en los caninos. En pequeños rumiantes y en ocasiones también en la vaca, recibe a la vena occipital, luego de su formación (Ghoshal et al., 1981). 
En el dromedario la cabeza y el cuello, son drenados por la vena yugular externa; la vena yugular interna, es una vaso pequeño que drena exclusivamente algunas estructuras del cuello. La vena yugular externa en esta especie está formada por la confluencia de las venas linguofacial y maxilar. La vena linguofacial, drena las estructuras de la cara, vía la vena facial y las estructuras de la cavidad oral, vía la vena lingual (Smuts \& Bezuidenhout, 1987).

De la descripción realizada por Khidr (1980), se extrae el siguiente párrafo: "la yugular está representada exclusivamente por la vena yugular externa, y no se observó la presencia de la vena yugular interna en ninguno de los ejemplares disecados. La yugular externa está formada por la unión de las venas facial y maxilar, unos $5 \mathrm{~cm}$ en craneal de la terminación del ala del atlas, en posición normal de estación. Está cubierta por la glándula parótida y relacionada profundamente con el tendón de la porción mastoídea del músculo esternocefálico. En el tercio proximal del cuello, se ubica subcutáneamente. También la cubren, la fascia superficial y el músculo cutáneo del cuello, hasta la altura de la tercera o cuarta articulación intervertebral. Caudalmente, la vena se profundiza quedando cubierta por el músculo esternomastoídeo ventralmente y dorsalmente por el músculo omotransverso. En los dos tercios distales del cuello, el nervio laríngeo se pone en contacto con su superficie medial y la arteria carótida común, se ubica en el lado dorsomedial. Las dos venas yugulares se unen justo antes de la entrada al tórax formando un corto tronco venoso de aproximadamente uno o dos centímetros ubicado sobre la cara dorsal de la tráquea; confluencia yugular según Chauveaux \& Arloing (1891) o yugular común. Esta confluencia recibe las venas cefálica y axilar para constituir la vena cava anterior".

Al referirse a las venas intervertebrales, la Nomina Anatomica Veterinaria (NAV, 1994) aclara: "el término de venas intervertebrales designa a las venas que pasan a través de los forámenes intervertebrales. Éstas conectan los plexos vertebrales externo e interno. El plexo interno es el antiguamente denominado seno vertebral. Éstas dan origen a los ramos interarcuales, los cuales penetran en los ligamentos amarillos; los ramos espinales, unen las venas espinales ". Como ramas derivadas de la vena intervertebral, cita la NAV, a los plexos vertebrales externo ventral, externo dorsal e interno ventral, derivando de este último, los ramos interarcuales y los espinales. Los plexos venosos externos dorsal y ventral, se sitúan en relación con las superficies externas de las láminas de los arcos vertebrales y de las caras ventrales de los cuerpos vertebrales, respectivamente (Schaller, 1996).

Smuts, dice respecto a las venas intervertebrales de esta especie, al citarlas como ramas de la vena vertebral: "en cada segmento, desde la segunda a la sexta, de la vena vertebral, se separa una vena intervertebral. Esta ingresa por el foramen intervertebral y se une al plexo venoso vertebral interno ventral. Éste puede originarse directamente de la vertebral o, más frecuentemente, en común con el ramo dorsal. La vena intervertebral es, a menudo, doble o puede dividirse en 2 a 4 ramos intercomunicantes para formar un pequeño plexo. Caudalmente al sexto espacio, corre a lo largo de la incisura vertebral caudal de $\mathrm{C} 6$ antes de unirse al plexo venoso vertebral interno ventral. La vena está además conectada al plexo en el ángulo de divergencia de las venas vertebral y cervical profunda".

A las características morfológicas que se han citado, se suman las hemodinámicas de los camélidos sudamericanos, enunciadas por Vallenas (1991):

- Elevado número de glóbulos rojos, de tamaño pequeño y forma elíptica.

- Una vida media de los eritrocitos que llega a los 60 días.

- Elevada concentración de hemoglobina corpuscular media [HbCM].

- Elevada tasa de miohemoglobina.

- Moderada elevación de la presión pulmonar en la altura, la cual no genera una consecuente hipertrofia del ventrículo derecho, ni engrosamiento de las paredes arteriales (ciertos autores indican contrariamente que se manifiesta un aumento de la capa media de las arterias en cuestión).

- Gran afinidad de la hemoglobina por el oxígeno, desplazamientos hacia la izquierda de la curva de disociación de la oxihemoglobina, estudio realizado en la alpaca a 3300 metros sobre el nivel del mar.

Si bien no concluye sobre los mecanismos específicos por los cuales los camélidos sudamericanos se adaptarían mejor a las alturas que otros mamíferos, se especula sobre la alta afinidad que tendría la hemoglobina por el oxígeno, sumado a ciertas particularidades de los tejidos.

\section{MATERIAL Y MÉTODO}

Las disecciones se realizaron en diez llamas provenientes de la localidad de González Moreno, Provincia de La Pampa, República Argentina. Todos los ejemplares eran machos, adultos y de aptitud lanera y en ellos se siguió el siguiente protocolo:

- Premedicación, entre 1,5 y 2,5 cc. de xilazina (Rompún ${ }^{\circledR}$ Lab. Bayer), por inyección intramuscular en la región femoral caudal.

- Inyección endovenosa de Pentothal Sódico®1g, Lab. 
Abbott, diluido en $5 \mathrm{cc}$ de agua.

- Alcanzado el plano anestésico profundo, se procede al sangrado "a blanco".

Se procedió luego a la inyección endovenosa de gelatina al $17 \%$, coloreada con anilina azul marino, canalizando la vena nasal dorsal o la vena facial. La inyección finaliza cuando se hace evidente la repleción de la vena yugular externa mediante la palpación del vaso en su recorrido cervical.

Durante las diferentes etapas de disección, los especímenes fueron pulverizados con una solución conservadora de base acuosa, conteniendo formol al 7\%, ácido fénico al $10 \%$, alcohol etílico $15 \%$, glicerina $20 \%$ y propilenglicol $15 \%$. Posteriormente, los preparados se cubrieron con film transparente y con la propia piel del animal $\mathrm{y}$, de esta manera, se guardaron en cámara frigorífica a $5^{\circ} \mathrm{C}$.

Las disecciones se realizaron con técnicas de disección e instrumental convencionales. Para los detalles anatómicos se utilizó una lupa estereoscópica con luz incorporada.

\section{RESULTADOS}

En los especímenes disecados, se observó que la vena yugular externa está formada por la confluencia de las venas lingual y maxilar. En su trayectoria hacia caudal por la región cervical, la vena yugular externa recibe como afluentes tres o cuatro venas provenientes del interior del canal vertebral, las cuales emergen a través de los forámenes intervertebrales. Estos delicados vasos se originan de la vena vertebral. Esta última se forma por varias ramas en dorsal del atlas, pasa por el foramen transverso y luego atraviesa un orificio ubicado en ventral del agujero vertebral lateral del axis para ingresar por la incisura craneal de esta vértebra al canal vertebral.

Esta disposición se debe a que, excepto del atlas, el resto de las vértebras cervicales de la llama no poseen foramen transverso en relación a la apófisis homónima, existiendo en su reemplazo un canal que recorre la parte craneal de la superficie medial del pedículo vertebral, razón por la cual se propone la denominación de "canal pedicular cervical". En él la vena vertebral se dirige caudalmente acompañada por la arteria homónima. En su recorrido colecta la sangre de las vértebras, meninges y médula espinal. Al llegar al foramen intervertebral entre $6^{\mathrm{a}}$ y $7^{\mathrm{a}}$ cervicales, la vena vertebral abandona el canal para ubicarse en ventral del proceso transverso de la última vértebra cervical. Sigue su curso por medial de la primera costilla y del origen del plexo braquial, para abrirse en el tronco costocervical del lado derecho y directamente en la v. cava craneal del lado izquierdo.

Las venas intervertebrales, una o más frecuentemente dos por foramen intervertebral, se muestran como el nexo entre la v. vertebral y la v. yugular externa. Se relacionan con la superficie lateral de la arteria vertebral, en los espacios ubicados entre las incisuras vertebral craneal y caudal de dos vértebras contiguas. Una vez formadas, siguen un curso flexuoso, sobre la cara lateral de la vértebra, cubiertas por los músculos intertransversos ventrales cervicales. Pasan por craneal de las apófisis transversas, para dirigirse caudoventralmente en busca de su desembocadura en la vena yugular externa.
Fig. 1. Esquema del circuito venoso cervical de la llama: 1- vena yugular externa; 2vena vertebral; 3- vena occipital; 4- arcos venosos cervicales. Con círculos se indica la posición de las vv. intervertebrales. 


\section{DISCUSIÓN}

Los resultados obtenidos demuestran la existencia de un sistema venoso cervical, comparable al descrito en las especies domésticas. Sin embargo, en la llama la vena vertebral cumple el rol de los plexos venosos vertebrales y las venas intervertebrales hacen las veces de puente o nexo entre aquella y la vena yugular externa, por lo cual es posible referirse a un verdadero circuito venoso cervical, favorecedor del retorno venoso.

Se concluye que existe un verdadero circuito verte- bral - yugular, que cumple la función de una vía alternativa para que la sangre acumulada en el territorio venoso de la cabeza, en los momentos en que el animal pastorea, tenga un rápido retorno hacia el corazón cuando el animal eleva el cuello, quedando este importante volumen sanguíneo disponible para ser redistribuido hacia otros órganos. Así, a las variaciones fisiológicas hemodinámicas conocidas para esta especie, se le suman en forma concomitante particularidades morfológicas que favorecen su adaptación a la altura.

ARZONE, C. A.; SÁNCHEZ, G. A.; VIDAL FIGUEREDO, R. V.; ACERBO, M. \& PELLEGRINO, F. C. Cervical venous circuit of the llama (Lama glama). Int. J. Morphol.,26(4):849-852, 2008.

SUMMARY: The neck region of the Llama (Lama glama) has several structural and functional particularities in comparison to the same region of other domestic mammals. Among them, it is noticeable a distinctive venous circuit whose description is the purpose of this study.In order to perform the study, the veins of the neck of ten adult, male, wool type llamas were dissected with conventional technique. Previously to dissection, the veins were filled with a repletive substance.The authors describe the morphology of a venous circuit in which external jugular vein (v. jugularis externa), vertebral vein (v. vertebralis), and intervertebral veins (vv. Intervertebrales) are the main participating vessels. The distinctive feature is the fact that the metameric anastomoses between the forenamed veins leads to a jugular-vertebral by pass. The existence of venous shunts provides a circuit of collateral circulation, diverting the blood mainly towards the external jugular vein. Grazing habits and the length of the neck suggest that the vascular structure previously described facilitates the venous return, contributing to the regional blood stream control.

KEY WORDS: Neck veins; Veterinary Anatomy; New World Camelids.

\section{REFERENCIAS BIBLIOGRÁFICAS}

Chaveaux, A. \& Arloing, S. Traité d'Anatomie comparée des animaux domestiques. $4^{\text {ème }}$ ed. J. B. Bailliere, París, 1891.

Ghoshal, N. G.; Koch, T. \& Popesko, P. The venous drainage of the domestic animals. W. B. Saunders Company, Philadelphia, 1981.

Khidr, I. M. S. The venous drainage of the head and neck of yhe one humped camel (Camelus dromedarius). Tesis presentada para el grado de PhD. (Anatomía). Faculty of Veterinary Medicine, Zagazig University, 1980.

Nómina Anatómica Veterinaria, WAVA. (Asociación Mundial de Anatomistas Veterinarios), $4^{\mathrm{a}}$ Ed. Zürich \& Ithaca, New York, 1994.

Schaller, O. Nomenclatura anatómica veterinaria ilustrada. Acribia, S.A., Zaragoza, España, 1996.

Smuts, M. M. S. \& Bezuidenhout, A. J. Anatomy of the Dromedary Clarendon. University Press, Oxford, 1987.
Vallenas, A. Características anatomofisiológicas (capítulo 2), en Fernandez Baca, S. (compilador): Avances y perspectivas del cconocimiento de los camélidos sudamericanos. Oficina Regional de la FAO para América Latina y el Caribe, 1991.

\author{
Dirección para correspondencia: \\ Dr. Carlos Arzone \\ Cátedra de Anatomía \\ Facultad de Ciencias Veterinarias \\ Avda. Chorroarin 280 \\ C1427CWO \\ Universidad de Buenos Aires \\ ARGENTINA
}

Email: carzone@fvet.uba.ar

Received: 15-07-2008

Accepted: 22-09-2008 